\title{
A Study on the Cultivation Strategies of Students' Scientific Research
}

\section{Thinking in Clinical Medicine}

\author{
ZHANG Zhuo ${ }^{1, a}$, JI Guoyi ${ }^{1, b}$, ZHANG Haiyan ${ }^{2, c}$, \\ ${ }^{1}$ Urology Department, China Japan Union Hospital of Jilin University, Changchun City, Jilin \\ Province, China, 130033 \\ ${ }^{2}$ Urology Department, China Japan Union Hospital of Jilin University, Changchun City, Jilin \\ Province, China, 130033 \\ ${ }^{3}$ Gastrointestinal Department, China Japan Union Hospital of Jilin University, Changchun City, \\ Jilin Province, China, 130033 \\ Corresponding Author: ZHANG Haiyan,3455064@qq.com
}

Keywords: Cultivation Strategy, Student, Scientific Research Thinking, Clinical Medicine

\begin{abstract}
With the gradual improvement for the abilities of medical professionals, the clinical medicine education can not meet the demands of the society for the medical workers in the new era. Therefore, based on the author's work experience, this paper discusses the training experience of clinical medical students' scientific research thinking. This paper first analyzes the factors that affect the scientific research thinking of clinical medicine students, and then put forward the concrete strategies training students' scientific research thinking, hoping to help students form the correct scientific outlook and values and to improve their own scientific research quality.
\end{abstract}

\section{Introduction}

Scientific research activity is a process of exploring, studying and creating with scientific knowledge, and it is also an important form for students to use scientific knowledge in the research and practice. Students of clinical medicine is a main force in achieving clinic scientific and technological innovation, so strengthening the cultivation of students' scientific research ability in clinical medicine is of great significance to improve the level of medical science and technology.

\section{Factors Affecting the Scientific Research Thinking of Clinical Medical Students}

Factors of teaching methods. Traditional teaching methods ignore the cultivation of students' creative ability, and most students are still accustomed to the learning of the existing book knowledge. Restricted by major, not many students have access to cross subject knowledge, so the depth, breadth and accuracy of knowledge are obviously inadequate. The knowledge structure which is not perfect enough weakens the students' ability to reflect and independent personality development, so it is difficult to stimulate students' sense of innovation.

Reasons from teachers. Clinical medicine teachers are responsible for the teaching of clinical knowledge and skills, guiding scientific research activities, guiding the thesis and thesis writing, etc., so the tutor plays a guider role from first to last. On the cultivation of scientific research ability of medical students, the teacher has an irreplaceable role. But instructors are often engages in clinical, teaching and scientific research, they rarely have the opportunity to explore with students, 
ignoring the subjectivity of students learning, and it is difficult to stimulate students' enthusiasm for innovation.

Students' own factors. Due to the following reasons, clinical medical students' scientific research ability is weak. First, lack of scientific research desire. Many clinical medical students often meet the status quo and give up the pursuit due to the heavy clinical work. Second, lack of time to engage in scientific research. Clinical medical students' learning burden is heavy, and the time is relatively tight, when engaging in heavy clinical work, they have to go through all kinds of medical tests, so there is a lack of time to do research. Third, lack of scientific research required observation. In the observation of the speed and breadth, integrity and generality, planning and flexibility, clinical medical students are generally inadequate. Forth, lack of scientific thinking ability. Most of the clinical medical students is still relatively immature in the intuitive thinking ability, logical thinking ability, thinking ability, divergent thinking, reverse thinking ability etc.

Medical social functions dominated by health care causes the scientific research consciousness is not strong. Under the current market economy, people's legal awareness is enhanced, and a little careless will lead to medical accidents and errors, so many doctors believe that it is enough as long as they can do a good job of daily work, improve service attitude, and do not have errors. At the same time, for a long time, due to the lack of preparation, the daily heavy work has made them in a state of overload, so the medical staff have little time and energy to carry out clinical research work.

\section{Cultivation Strategies of Scientific Research Thinking for Clinical Medical Students}

To strengthen the systematic training of students' scientific research ability. Scientific research ability is a comprehensive ability. Teachers should strengthen guidance and enlightenment for students' scientific research ability, and during the preliminary work of scientific research, they should put forward reasonable proposals and related matters of attention to students. In combination with the practical system, the experiment ability was cultured when the research work is carried out, including the ability to access and process the literature, experimental design, experimental operation, the summary of the experiment and paper writing, etc.. Under the guidance of teachers, review literature - write a summary - write vertical title - design experiments - complete the experimental operation - finish data - write the paper, every link must be carefully taught. Therefore, teachers should carry out education and training programs based on the actual situation of students, so that students feel that the content of the teaching content is practical, and is closely related to their own, is concerned about their own growth and progress.

To strengthen the professional knowledge and basic skills training to lay a solid foundation for the cultivation of scientific research ability. Medicine is a practical medicine, and learning professional knowledge is the most important task. Scientific research is only the use and strengthening of professional knowledge, and the actual medical work is the main source of the research project, as long as paying attention to observation, it can gradually form a topic to explore. Therefore, clinical medical students should be combined with clinical research activities, rather than separate from clinical independent research activities. When training students to use the knowledge to solve clinical problems, it not only can mobilize the initiative and enthusiasm of the students, but also can cultivate the students' interest in scientific research.

Paying equal attention to the cultivation of comprehensive ability to achieve both knowledge and ability. Scientific research ability is a higher level of ability, which needs a variety of basic ability for the protection.If one person wants to really have strong scientific research ability, 
he or she must have a strong comprehensive ability and comprehensive quality, especially the observation ability, the ability to find and ask questions, logical thinking ability, creative ability, writing ability, analysis and problem solving ability, independent thinking ability, learning ability and the ability of summing, which depends on constant thinking and learning to get. Clinical medicine students need to do the following: First, good at finding problems and asking questions; Second, good at analysis and thinking with the courage to explore; Third, paying attention to cultivate their own abilities of scientific and rational classification and summary.

Training students to access the information to obtain the effective information. To carry out scientific research activities, we must first master a lot of information about the object of study, understand the latest research trends of the international and domestic counterparts and the research contents and results in this field, to determine the academic status and value of the research they are about to engage in. Scientific research should make innovative work, put forward their own point of view, design their own experimental program. Therefore, it is necessary to make a full use of network resources to get the relevant information and make new exploration and breakthrough on the basis of the existing research of other people. Only in this way, can they avoid duplication of research, and make their research activities have a higher starting point.

Paying more attention to English learning. English is one of the main international languages in the world, and it is also the most widely used language in the world. There are about 20 countries in the world to use English as the official language or the second language. More than $70 \%$ of the world's scientific and technical publications are published in English. The vast majority of international conferences uses English as the first language. In short, English is an important tool for communication. Medical students need to consult a large number of foreign materials in the process of scientific research. To cultivate students' interest and enthusiasm in foreign language learning and to improve the level of foreign language as soon as possible will be of great help to the future research work. Therefore, students should be encouraged to use the Internet platform to learn English, to improve the ability of English listening and speaking. At the same time, it needs to encourage students to actively participate in the academic report organized by the school, domestic and foreign experts. Encouraging students to actively communicate and actively participate can create a platform for students to show the overall quality, so that their innovative awareness will be strengthened.

Guiding students to write scientific papers. The publication of scientific research papers is the affirmation of their research work. Medical students need to actively write papers for publication in the Journal of science and technology, including the domestic core journals and SCI journals. In particular, medical students should be encouraged to publish their papers in the Journal SCI. SCI journal is an important academic journals in the global natural sciences, and it is the main medium of communication in the international academic circles. Only papers published in the Journal SCI can be known to the international counterparts. Therefore, on the basis of efforts to improve the level of English, clinical medical students should read a large number of SCI papers, master the writing method of SCI papers, exercise the ability to write SCI papers.

Cultivating students' scientific attitudes. In the actual operation of scientific research, it will encounter a variety of difficulties. When encountering difficulties and setbacks, it needs to actively analyze the causes of the problem, to figure out ways to solve the problem. In the process of scientific research, training scientific research thinking, the ability to solve practical problems. As a student, they must have a strict attitude, must strictly observe the occupation ethics to carry out careful logical analysis, rigorous test design and strict test operation. 


\section{Conclusion}

The cultivation of students' scientific research ability in clinical medicine is related to the sustainable development of medical research and medical treatment, and the scientific research activities can make the students have a strong incentive to innovate, set up the correct goal of innovation and give full play to the potential of innovation and ingenuity, therefore, to strengthen the cultivation of students' scientific research ability is not only the demand of the development of medical career, but also the demand of the development of information society.

\section{References}

[1] Shi Weiwei, Ren Yi, Gu Yixing, Cheng Xiaoyan. The cultivation of scientific research thinking and innovative spirit of the professional degree of clinical medicine in the hospital [J]. Chinese Hospital, 2011,10:65-67.

[2] Luo Gengqiu, Wen Jifang,, Zhou Jianhua. Research on the cultivation of scientific thinking ability of eight year medical students in clinical medicine [J]. Journal of Shanxi Medical University (basic medical education), 2009,01:49-50.

[3] Wang Yajuan, Lin Ying, Meng Xiaoying. Strengthening the training of scientific research ability of students in clinical medicine specialty [J]. Journal of Capital Medical University (SOCIAL SCIENCE EDITION), 2010,00:470-472.

[4] Shi Guangli, Chen Yan. The cultivation of students' scientific research ability of medical laboratory science [J]. Continuing Medical Education, 2016,02:15-16.

[5] Wang Dong, Li Hongxing, Zhang Lianshuang. Thinking on the cultivation of scientific research thinking for clinical medical undergraduates [J]. Contemporary Educational Practice and Teaching Research, 2014,12:91-92. 